\title{
Representations of Edge Intersection Graphs of Paths in a Tree
}

\author{
Martin Charles Golumbic ${ }^{1 \dagger}$, Marina Lipshteyn ${ }^{1}$ and Michal Stern ${ }^{1}$ \\ ${ }^{1}$ Caesarea Rothschild Institute, University of Haifa, Haifa, Israel
}

Let $\mathcal{P}$ be a collection of nontrivial simple paths in a tree $T$. The edge intersection graph of $\mathcal{P}$, denoted by $\operatorname{EPT}(\mathcal{P})$, has vertex set that corresponds to the members of $\mathcal{P}$, and two vertices are joined by an edge if the corresponding members of $\mathcal{P}$ share a common edge in $T$. An undirected graph $G$ is called an edge intersection graph of paths in a tree, if $G=E P T(\mathcal{P})$ for some $\mathcal{P}$ and $T$. The EPT graphs are useful in network applications. Scheduling undirected calls in a tree or assigning wavelengths to virtual connections in an optical tree network are equivalent to coloring its EPT graph.

It is known that recognition and coloring of EPT graphs are NP-complete problems. However, the EPT graphs restricted to host trees of vertex degree 3 are precisely the chordal EPT graphs, and therefore can be colored in polynomial time complexity. We prove a new analogous result that weakly chordal EPT graphs are precisely the EPT graphs with host tree restricted to degree 4. This also implies that the coloring of the edge intersection graph of paths in a degree 4 tree is polynomial.

We raise a number of intriguing conjectures regarding related families of graphs.

Keywords: Paths of a tree, Intersection graphs, Weakly chordal graphs, Coloring, EPT-graphs

\section{Introduction}

All standard definitions of terms we use can be found in $[4,15]$.

Let $\mathcal{P}$ be a collection of nontrivial simple paths in a tree $T$. We define two different types of intersection graphs from the pair $\langle\mathcal{P}, T\rangle$, namely the VPT and $k$-EPT graphs. The vertex intersection graph $V P T(\mathcal{P})$ of $\mathcal{P}$ has vertices which correspond to the members of $\mathcal{P}$, such that two vertices are adjacent in $V P T(\mathcal{P})$ if the corresponding paths in $\mathcal{P}$ share a vertex in $T$. An undirected graph $G$ is called $a$ vertex intersection graph of paths in a tree (VPT) if $G=V P T(\mathcal{P})$ for some $\mathcal{P}$ and $T$, and $<\mathcal{P}, T>$ is a VPT representation of $G$. Similarly, we define the $k$-edge $(k \geq 1)$ intersection graph $k$-EPT $(\mathcal{P})$ of $\mathcal{P}$ to have vertices which correspond to the members of $\mathcal{P}$, such that two vertices are adjacent in $k$-EPT $(\mathcal{P})$ if the corresponding paths in $\mathcal{P}$ share $k$ edges in $T$. An undirected graph $G$ is called a k-edge intersection graph of paths in a tree $(k$-EPT) if $G=k$-EPT $(\mathcal{P})$ for some $\mathcal{P}$ and $T$, and $\langle\mathcal{P}, T\rangle$ is a $k$-EPT representation of $G$. The case of $k=1$ (known as the EPT graphs) was introduced by Golumbic and Jamison [5, 6]. Finally, we denote by EPT* the class of graphs consisting of the $k$-EPT graphs for all possible $k \geq 1$.

\footnotetext{
†golumbic@cs.haifa.ac.il

1365-8050 @ 2005 Discrete Mathematics and Theoretical Computer Science (DMTCS), Nancy, France
} 
The complexity of recognizing VPT graphs is polynomial [3], but recognition of 1-EPT [5], $k$-EPT and EPT* graphs $[7,8]$ is NP-complete.

Clearly, for a given collection $\mathcal{P}$, the vertices of $k-E P T(\mathcal{P})$ and those of $V P T(\mathcal{P})$ are the same, and the edge sets are nested: $E(k-E P T(\mathcal{P})) \subseteq E((k-1)-E P T(\mathcal{P})) \subseteq \ldots \subseteq E(1-E P T(\mathcal{P})) \subseteq E(V P T(\mathcal{P}))$. The following is also easy to prove.

Lemma 1.1 [8] Let $G$ be a 1-EPT graph, then $G$ is a $k$-EPT graph, for every $k \geq 2$.

The $k$-EPT graphs are used in network applications, where the problem of scheduling undirected calls in a tree network is equivalent to the problem of coloring a 1-EPT graph (see e.g. [2, 5]).

In [6], Golumbic and Jamison showed that the coloring problem of 1-EPT graphs is NP-complete and this was extended to $k$-EPT graphs in [7,8]. Tarjan gave a $\frac{3}{2}$-approximation algorithm for coloring 1EPT graphs [17]. However, when the maximum degree of the host tree $T$ is 3 , the coloring problem is polynomial. We show the analogous polynomial result for a degree 4 host tree.

Theorem 1.2 [5] Let $G$ be an undirected graph. The following statements are equivalent:

(i) $G$ is both a VPT graph and a 1-EPT graph.

(ii) G has a VPT representation on a degree 3 tree.

(iii) G has a 1-EPT representation on a degree 3 tree.

Theorem 1.3 [8] Let $G$ be a VPT graph. Then, $G$ is a 1-EPT graph if and only if $G$ is a k-EPT graph.

We denote by $C_{n}$ the chordless cycle on $n$ vertices. The chordless cycle $C_{n}$ is a 1-EPT graph and it has a unique 1-EPT representation which is called a pie in [6], defined as follows. Theorem 1.4 shows that this is essentially the only representation for $C_{n}$.

Let $\langle\mathcal{P}, T\rangle$ be a 1-EPT representation of a graph $G$. A pie is a star subgraph of $T$ with $n$ edges $\left(a_{0}, b\right), \ldots,\left(a_{n-1}, b\right)$ such that each "slice" $\left(a_{i}, b\right) \cup\left(a_{i+1}, b\right)$ for $i=0,1, \ldots, n-1$ is contained in a different member of $\mathcal{P}$, where addition is assumed to be modulo $n$. The vertex $b$ is center of the pie.

Theorem 1.4 [6] Let $<\mathcal{P}, T>$ be a 1-EPT representation of a graph $G$. If $G$ contains a chordless cycle of length $n \geq 4$, then $<\mathcal{P}, T>$ contains a pie with $n$ edges.

We note that Theorem 1.4 does not hold for larger values of $k$. In fact, in [8] we give representation of $C_{n}$ on a degree 3 tree, for $k \geq 2$ and $n \geq 4$.

Remark 1.5 [6] A 1-EPT graph contains no $\bar{C}_{n}, n \geq 7$. The 1-EPT representations of graphs $\bar{C}_{5}$ and $\bar{C}_{6}$ can be easily found.

An undirected graph $G$ is chordal if every cycle in $G$ of length strictly greater than 3 possesses a chord. It is well-known that chordal graphs are the vertex intersection graphs of subtrees of a tree. Therefore, every VPT graph is chordal. A graph $G$ is weakly chordal if neither $G$ nor its complement $\bar{G}$ have an induced subgraph $C_{k}, k \geq 5$. Weakly chordal graphs can be recognized and colored in polynomial time $[1,10,11]$. A two-pair in a graph $G$ is a pair of vertices $(x, y)$, such that every chordless path between $x$ and $y$ contains exactly two edges.

Theorem 1.6 [10] A graph $G$ is weakly chordal if and only if every induced subgraph of $G$ either has a two-pair or is a clique.

In [12,13], Jamison and Mulder define a $(h, s, p)$-representation, which consists of a collection of subtrees of a tree, such that (i) the maximum degree of $T$ is at most $h$, (ii) every subtree has maximum 
degree at most $s$, and (iii) there is an edge between two vertices in the graph if the corresponding subtrees in $T$ have at least $p$ vertices in common. A class of graphs that have a $(h, s, p)$-representation is denoted by $[h, s, p]$. Thus, the class of $k$-EPT graphs corresponds to the class $[\infty, 2, k+1]$ and the chordal graphs are $[\infty, \infty, 1]$.

Definition 1.7 A subtree $T^{\prime}$ of a tree $T$ is leaf-generated if all endpoints of $T^{\prime}$ are leaves in $T$. A representation $\langle\mathcal{T}, T>$ of a graph $G$ is leaf-generated if all the subtrees in $\mathcal{T}$ are leaf-generated subtrees of the host tree $T$. A representation $<\mathcal{T}, T>$ of a graph $G$ is orthodox if it is leaf-generated and two subtrees in $\mathcal{T}$ share a leaf in $T$ if and only if the corresponding vertices are adjacent in $G$.

The terminology of an orthodox representation is used in Jamison and Mulder[12, 13], who cite an earlier result of McMorris and Scheinerman [14], namely, $(i) \Leftrightarrow(i i) \Leftrightarrow($ iii $)$ in Theorem 1.8 below, and prove in [12] the remaining equalities.

Theorem $1.8[12,13,14]$ The following statements are equivalent:

(i) A graph $G$ is chordal,

(ii) G has a $(3,3,1)$-representation,

(iii) $G$ has an orthodox $(3,3,1)$-representation,

(iv) $G$ has a $(3,3,2)$-representation,

$(v) G$ has an orthodox $(3,3,2)$-representation.

\section{Weakly Chordal 1-EPT Graphs}

It is known that coloring of 1-EPT graphs is NP-complete problem. However, the 1-EPT graphs restricted to host trees of vertex degree 3 are precisely the chordal 1-EPT graphs, and therefore can be colored in polynomial time complexity. We prove a new analogous result that weakly chordal 1-EPT graphs are precisely the 1-EPT graphs with host tree restricted to degree 4 . This also implies that coloring an edge intersection graph of paths in a degree 4 tree is polynomial.

Lemma 2.1 A 1-EPT graph restricted to degree 4 trees contains no $\bar{C}_{5}$ and no $\bar{C}_{6}$.

We now present the main result of this section.

Theorem 2.2 A graph $G$ is weakly chordal and 1-EPT graph if and only if $G$ has a 1-EPT representation on a degree 4 tree.

Proof Sketch: $(\Leftarrow)$ Let $\langle\mathcal{P}, T\rangle$ be a 1-EPT representation of a graph $G$, where $T$ is of degree 4 . We must show that $G$ is weakly chordal. By Theorem 1.4, if $G$ contains a chordless cycle $C_{n}(n \geq 5)$, then $<\mathcal{P}, T>$ contains a pie with $n$ edges. Since $T$ is restricted to degree 4 , this is impossible. Therefore, $G$ has no $C_{n}(n \geq 5)$.

Moreover, $G$ contains no $\bar{C}_{n}, n \geq 7$ due to Remark 1.5 , and $G$ contains no $\bar{C}_{5}$ or $\bar{C}_{6}$ due to Lemma 2.1. It now follows that $G$ is weakly chordal.

$(\Rightarrow)$ Let $\langle\mathcal{P}, T\rangle$ be a 1-EPT representation of a weakly chordal graph $G$, where $T$ has maximal degree $d>4$. We now prove by induction on the number of vertices of degree $d$ that there exists a 1-EPT representation $\left\langle\mathcal{P}^{\prime}, T^{\prime}>\right.$ of $G$ with maximal degree $d^{\prime}$, where $d>d^{\prime} \geq 4$.

Let $u$ be a vertex of degree $d$ in $T$ with neighbors $v_{1}, \ldots, v_{d}$. Let $\mathcal{P}_{U}$ be the collection of paths of $\mathcal{P}$ that contain at least one of the edges $\left(v_{1}, u\right), \ldots,\left(v_{d}, u\right)$ in $T$. Let $G_{U}$ be the induced subgraph of $G$, such 
that every vertex in $G_{U}$ corresponds to a path in $\mathcal{P}_{U}$. By the hereditary property, the induced subgraph $G_{U}$ is also a 1-EPT graph.

According to Theorem 1.6, the subgraph $G_{U}$ is either a clique or has a two-pair. We consider these two cases independently. In each of the cases, we obtain $\left\langle\mathcal{P}^{\prime}, T^{\prime}\right\rangle$ from $\langle\mathcal{P}, T\rangle$ by changing the representation, such that the number of vertices of degree $d$ does not increase and the degree of $u$ is decreased. Therefore, $\left\langle\mathcal{P}^{\prime}, T^{\prime}\right\rangle$ is a 1-EPT representation of $G$ with fewer vertices of degree $d$. By induction, there exists a 1-EPT representation $\left\langle\mathcal{P}^{\prime \prime}, T^{\prime \prime}>\right.$ of $G$ with maximal degree $d^{\prime}$, where $d>d^{\prime} \geq 4$.

We iteratively apply this construction to obtain a representation $\left\langle\mathcal{P}^{\prime}, T^{\prime}>\right.$ of $G$, where $T^{\prime}$ has a maximal degree 4 .

By using essentially the same arguments as in the proof of Theorem 2.2, we can obtain an alternate proof of the following:

Corollary 2.3 [16] A chordal graph $G$ is 1-EPT if and only if $G$ has a 1-EPT representation on a degree 3 tree.

Remark 2.4 The graph H shown in Appendix 4 is weakly chordal, but is not a 1-EPT graph.

\section{Further research}

An annoying open question remains regarding the relationship between the families of $k$-EPT and $(k+1)$ EPT graphs. In [12], Jamison and Mulder have conjectured that $k$-EPT $\subseteq(k+1)$-EPT and we have shown that the graph $F_{k}$, shown in Appendix 4, is $(k+1)$-EPT but is not $k$-EPT. Thus, their conjecture reduces to whether there is a proper containment between the two families. In this direction, they have also shown a weaker result that $k$-EPT $\subseteq k^{\prime}$-EPT for $k^{\prime} \geq k^{2}-2 k+2$.

A related open problem is to characterize the class of EPT* graphs. The figure in Appendix 4 shows examples of graphs which are not EPT*.

Several questions are raised by considering the restriction of the degree $h$ of the host tree. We have proved that the graphs $F_{1}$ and $D$ shown in Appendix 4 have a $k$-EPT representation restricted to degree 3 trees, but have no 1-EPT representation. Therefore, when restricted to degree 3 trees, the family of 1 EPT graphs is strictly contained in $k$-EPT graphs. We have also seen that the coloring problem on 1-EPT graphs is polynomial for $h=3$ or $h=4$. What is the complexity for higher values of $h$ ?

If a graph $G$ has a 1-EPT representation on a degree $h$ tree, then $G$ has no chordless cycle $C_{d}$ for $d \geq h+1$, since there is a unique 1-EPT representation of $C_{d}$ as a pie with $d$ slices. The converse is true for $h=3$, since $G$ must be chordal. The converse is false for $h=4$, since the graph $\bar{C}_{6}$ has no chordless cycle greater than 4 and does not have a 1-EPT representation on a degree 4 tree, by Lemma 2.1. It is easy to show that $\bar{C}_{6}$ has a 1-EPT representation on a degree 5 tree. We therefore ask the question, is the converse true for $h \geq 5$ ?

Just as $C_{d}$ must have degree $d$ in a 1-EPT representation, we have found a graph $W_{d}$ that must have degree at least $k+3$ in a $k$-EPT representation, for any fixed $k>1$. The graph $W_{d}$, for an integer $d>1$, is the graph with vertex set $V\left(W_{d}\right)=\left\{x_{i j} \mid 1 \leq i<j \leq d\right\}$ and edges $E\left(W_{d}\right)=\left\{\left(x_{i j}, x_{i^{\prime} j^{\prime}}\right) \mid i=i^{\prime}\right.$ or $i=j^{\prime}$ or $j=i^{\prime}$ or $\left.j=j^{\prime}\right\}$. Does there exist a well-defined family of graphs that restrict the degree of the host tree? 


\section{References}

[1] A. Berry, J. Bordat, P. Heggernes, Recognizing weakly triangulated graphs by edge separability, Nordic Journal of Computing 7(3), Fall(2000), 164-177.

[2] T. Erlebach, K. Jansen, Scheduling of virtual connections in fast networks, Proc. of the 4th Parallel Systems and Algorithms Workshop (PASA'96) 1996, 13-32.

[3] F. Gavril, A recognition algorithm for the intersection graphs of paths in trees, Discrete Math. 23 (1978), 211-227.

[4] M.C. Golumbic, Algorithmic Graph Theory and Perfect Graphs, Second Edition, Annals of Discrete Math. 57, Elsevier (2004).

[5] M.C. Golumbic, R.E. Jamison, Edge and vertex intersection of paths in a tree, Discrete Mathematics 55 (1985), 151-159.

[6] M.C. Golumbic, R.E. Jamison, The edge intersection graphs of paths in a tree, Journal of Combinatorial Theory, Series B 38 (1985), 8-22.

[7] M.C. Golumbic, M. Lipshteyn, M. Stern, The recognition of k-EPT graphs, Congressus Numernatium 171 (2004), 129-139.

[8] M.C. Golumbic, M. Lipshteyn, M. Stern, The k-edge intersection graphs of paths in a tree, submitted.

[9] M.C. Golumbic, M. Lipshteyn, M. Stern, The complete hierarchy of VPT, $k$-EPT and chordal graphs, abstract accepted for presentation at CTW05.

[10] R.B. Hayward, C.T. Hoàng, F. Maffray, Optimizing weakly triangulated graphs, Graphs and Combinatorcs 5, 339-349, 1989.

[11] R.B. Hayward, J. Spinrad, R. Sritharan, Weakly chordal graph algorithms via handles, Proceedings of the 11th ACM-SIAM Symposium on Discrete Algorithms (2000), 42-49.

[12] R.E. Jamison, H.M. Mulder, Constant tolerance intersection graphs of subtrees of a tree, Discrete Math. 290 (2005), 27-46.

[13] R.E. Jamison, H.M. Mulder, Tolerance intersection graphs on binary trees with constant tolerance 3, Discrete Math. 215 (2000), 115-131.

[14] F.R. McMorris, E. Scheinerman, Connectivity threshold for random chordal graphs, Graphs and Combin. 7 (1991), 177-181.

[15] J. Spinrad, Efficient Graph Representations, Providence, R.I.: American Mathematical Society (2003).

[16] M.M. Syslo, Triangulated edge intersection graphs of paths in a tree, Discrete Math. 55 (1985), 217-220.

[17] R.E. Tarjan, Decomposition by clique separators, Discrete Math. 55 (1985), 221-232. 


\section{Appendix: Hierarchy}

We prove in [9] that the hierarchy is complete, meaning that all containment relationships are given. That is, (1) classes that appear in the same box are equivalent, (2) a downward edge from class A to class B indicates that class A contains class B, (3) an example appearing along the edge between two classes is a separating example for those classes, (4) the lack of a hierarchical (containment) relation indicates that the classes are incomparable.

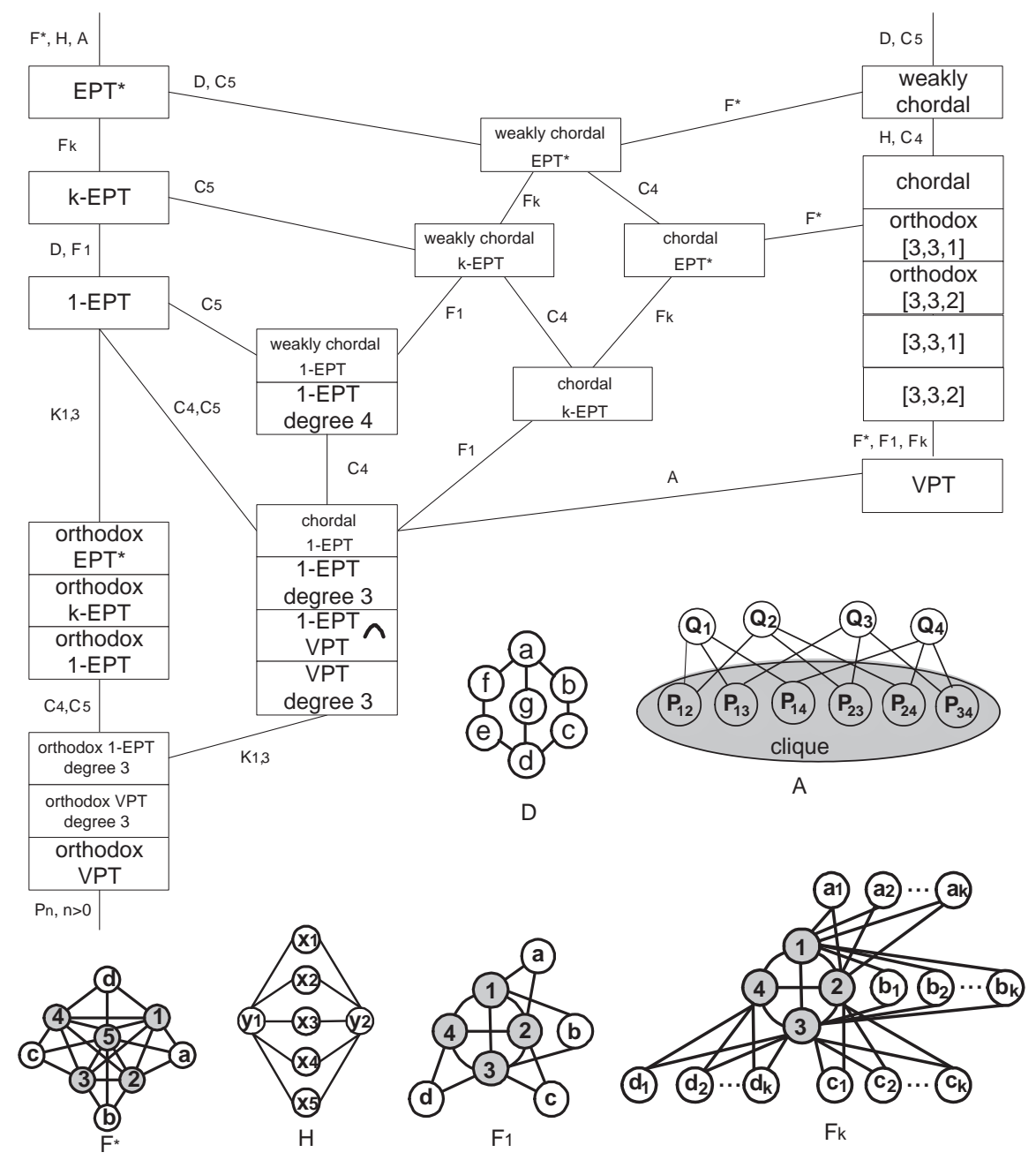

